\title{
A COMPARATIVE STUDY ON QUALITY ASSESSMENT OF HIGH RESOLUTION FINGERPRINT IMAGES
}

\author{
Qijun Zhao, Feng Liu, Lei Zhang, and David Zhang
}

Biometrics Research Centre, The Hong Kong Polytechnic University, Kowloon, Hong Kong

\begin{abstract}
High resolution fingerprint images have been increasingly used in fingerprint recognition. They can provide more fine features (e.g. pores) than standard fingerprint images to improve the recognition accuracy. It is however still an open issue whether or not existing quality assessment methods are suitable for high resolution fingerprint images. This paper compares some typical quality indexes by analyzing the correlation between them and their prediction ability on minutia-based and pore-based high resolution fingerprint recognition accuracy. Experimental results show that the indexes based on ridge orientation are more effective for high resolution fingerprint recognition systems.
\end{abstract}

Index Terms - Fingerprint Recognition, Quality Assessment, High Resolution Fingerprint Images

\section{INTRODUCTION}

Fingerprint recognition is one of the most widely used biometric techniques for personal authentication. Usually, two fingerprint images are not compared directly, but have the features on them extracted and matched. Whereas level1 and level-2 features such as singular points and minutiae (i.e. ridge endings and bifurcations) are the basis of most existing automatic fingerprint recognition systems (AFRS) [1], level-3 features such as pores are also very distinctive and are now increasingly used to improve fingerprint recognition accuracy, thanks to the advent of high resolution fingerprint imaging devices which enable the reliable extraction of them from fingerprint images [2-3].

When capturing fingerprint images, a number of factors can affect the image quality, e.g. the sensor noise, the acquisition conditions, and the finger skin conditions [1]. Fig. 1 shows two example fingerprint images, one of good quality and the other of poor quality. Features on the poor quality fingerprint images are difficult to precisely detect, and automatic algorithms often miss true features or detect spurious features. The recognition accuracy of AFRS will be consequently degraded. Therefore, in order to ensure good performance of AFRS, it is very important to evaluate the

This work is partially supported by The Hong Kong Polytechnic University Internal Fund (A-SA08). The authors would like to thank the anonymous reviewers. quality of captured fingerprint images. According to the quality of fingerprint images, appropriate follow-up operations can be then taken, e.g. re-capturing the fingerprint if the image quality is too bad, or incorporating the quality into feature extraction and matching to augment the recognition accuracy [4]. Many different methods have been proposed in the literature for assessing the quality of fingerprint images [5-10]. In Section 2, we will briefly review these methods.

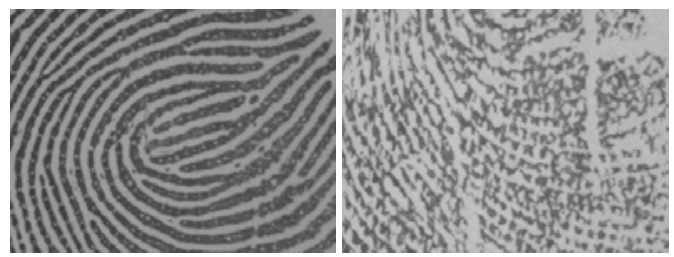

Fig. 1: One good quality (left) and one bad quality (right) high resolution fingerprint images.

High resolution ( $\geq 1000 \mathrm{ppi}$ ) fingerprint images have been recently increasingly used in fingerprint recognition. Compared with conventional low resolution ( 500ppi) fingerprint images, they provide more fine features on fingerprint ridges (e.g. pores) and can thus help to further improve the fingerprint recognition accuracy [2]. Because of the introducing of new features, it is unclear whether existing quality assessment methods are suitable for high resolution fingerprint images or not. So far, no study has been published on the quality assessment of high resolution fingerprint images. In this paper, we will investigate which method is better for evaluating the quality of high resolution fingerprint images when pores are utilized for fingerprint recognition. Section 3 will report and analyse the experimental results. Section 4 finally concludes the paper.

\section{QUALITY ASSESSMENT METHODS}

Existing fingerprint image quality assessment methods can be divided into three categories [10]. The first kind of methods are based on local features of fingerprint images, e.g. local ridge orientation and local ridge clarity. These methods are most widely used. They usually divide a fingerprint image into a number of blocks and extract features from each block. The quality of each block is then 
assessed based on the extracted features, and a quality index is finally calculated for the whole fingerprint image according to the quality of all the local blocks on it. A variety of local features have been exploited, including ridge orientation [6, 8], pixel intensity [7], Gabor features [5], and power spectrum [7].

Two typical local feature based fingerprint image quality indexes are orientation certainty level (OCL) [6] and uniformity of local pixel intensity [7]. The OCL index measures the energy concentration along the dominant ridge orientation on a local block. It can be calculated based on the eigenvalues of local structure tensor defined as

$J=\frac{1}{|B|} \sum_{i \in B}\left(g_{i}^{X}, g_{i}^{y}\right)^{T}\left(g_{i}^{X}, g_{i}^{y}\right)$

where $B$ is a local block and $|B|$ denotes the number of pixels on $B, g_{i}^{x}$ and $g_{i}^{y}$ are respectively the $\mathrm{x}$ - and $\mathrm{y}$ gradients at the pixel $i$, and ' $T$ ' is the transpose operator. Let $\lambda_{1}$ and $\lambda_{2}\left(\lambda_{1} \leq \lambda_{2}\right)$ be the two eigenvalues. The OCL index in [6] is then defined as $\lambda_{1} / \lambda_{2}$. Differently, the uniformity index evaluates the fingerprint image quality based on the degree to which similar pixels (i.e. ridges and valleys) cluster in the nearby region. To get the index value for a fingerprint image, the image is first binarized and the clustering factor is then calculated as the uniformity index (denoted as CFU).

Methods in the second category assess the fingerprint image quality based on global features. They analyze fingerprint images in a holistic manner. For example, in [6], the authors consider the continuity of fingerprint ridge orientation field and the uniformity of fingerprint ridge frequency field over the fingerprint image. The method in [8] is based on the observation that the ridge frequency values on a fingerprint image lie within a certain range. It thus assumes that the energy on the spectrum becomes more concentrated in few bands as the quality of fingerprint image increases. Based on this assumption, the method defines a global quality index which measures the energy concentration by using entropy (we denote this method as $\mathrm{EC})$.

The third kind of method predicts fingerprint image quality by using classifiers such as neural networks [9]. In these methods, the quality measure is essentially defined as a degree of separation between the match and non-match score distributions of a given fingerprint based on some fingerprint features (e.g. minutiae). They classify fingerprint images into five classes of image quality, instead of generating continuous quality index values for them.

From the above discussion, we can see that different quality indexes evaluate the fingerprint image quality from different aspects. As a result, they may be more effective in some cases, but less in others. Moreover, because the recognition performance of AFRS highly depends on the extraction and matching accuracy of the features used for fingerprint recognition, a good quality index for an AFRS should correlate with the features used by the AFRS and thus be able to predict its performance. Motivated by this, we in this study investigate the effectiveness of existing fingerprint image quality assessment methods on high resolution fingerprint images when level-3 features (i.e. pores) are used for recognition, and aim to provide helpful guidance on choosing and designing quality indexes for high resolution fingerprint images. In the next section, we report the experiments and analyze the obtained results.

\section{EXPERIMENTS}

\subsection{Selected Quality Indexes}

We considered two local feature based quality indexes, OCL [6] and CFU [7], and one global feature based quality index, EC [8]. These three quality indexes assess the fingerprint image quality based on local ridge orientation consistency, local ridge and valley clarity, and concentration of ridge frequency field, respectively. In the experiments, all quality index values were normalized into the range between 0 and 1 , with 0 denoting the worst quality and 1 the best quality.

\subsection{Database and Protocol}

We collected a set of high resolution fingerprint images by using our custom-built $\sim 1200 p p i$ fingerprint image acquisition device. There are totally 1480 images from 148 fingers, each finger having five images captured in each of two sessions (about two weeks apart). The spatial size of the images is 640 pixels in width and 480 pixels in height.

The three quality indexes were calculated for each of the fingerprint images. The correlation between the quality indexes was then studied. In order to investigate the recognition accuracy prediction ability of the three quality indexes, we implemented two fingerprint matchers, one based on minutiae [11] and the other based on pores [1213]. By using each matcher, the following genuine and imposter matches were carried out. 1) Genuine matches: each of the fingerprint images in the second session was matched with all the fingerprint images of the same finger in the first session, resulting in 3,700 genuine match scores. 2) Imposter matches: the first fingerprint image of each finger in the second session was matched with the first fingerprint images of all the other fingers in the first session, resulting in 21,756 imposter match scores. Following the strategy in [8], we sorted all the fingerprint images according to their quality, and pruned the last $p$ percent poor quality fingerprint images. The match scores corresponding to these poor quality fingerprint images were removed and the equal error rate (EER) was then calculated based on the rest match scores. By investigating the EERs under different $p$ 's, we can understand the recognition accuracy prediction ability of the quality index. Next, we report and analyze the obtained experimental results.

\subsection{Experimental Results and Analysis}


Fig. 2 plots the values of the three quality indexes on the high resolution fingerprint images used in the experiments. The Pearson correlation between each two of the quality indexes is also given. From the results, we can see that the Pearson correlations between the three quality indexes are all below 0.5. This is because they are based on different fingerprint features, and moreover, the good quality of one kind of features does not necessarily guarantee the good quality of other kinds of features.
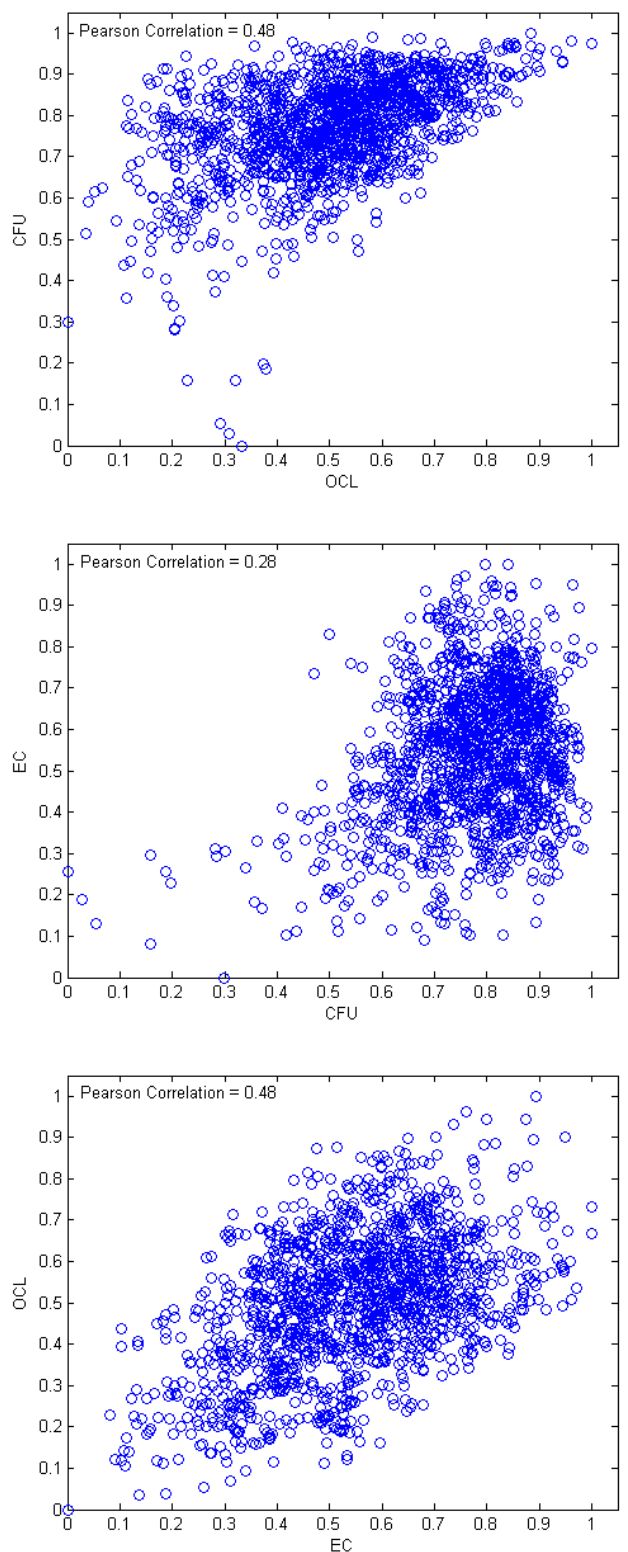

Fig. 2: Correlation between the three quality indexes on the high resolution fingerprint images used in the experiments.

The fingerprint recognition accuracy with respect to the quality of fingerprint images is shown in Fig. 3. We considered pruning 5\%, 10\%, 15\%, 20\%, 25\% and 30\% poor quality fingerprint images. If a quality index is good at predicting the recognition accuracy, the EER is expected to decrease as more poor quality fingerprint images are pruned according to the quality evaluated by the quality index. However, according to the results in Fig. 3, the CFU quality index does not work at all for either minutia-based or porebased fingerprint matchers on high resolution fingerprint images. This is better illustrated in Fig. 4. In the left fingerprint fragment, there are many pores (i.e. bright blobs) on ridges; whereas in the right fingerprint fragment, very few pores appear on ridges. By using the CFU quality index, the right fragment is evaluated to have better quality than the left one because the pores on ridges decrease the clarity of ridges. However, these pores are in fact useful features on high resolution fingerprint images. Consequently, the CFU quality index seems not suitable for quality assessment of high resolution fingerprint images as demonstrated in Fig. 3.
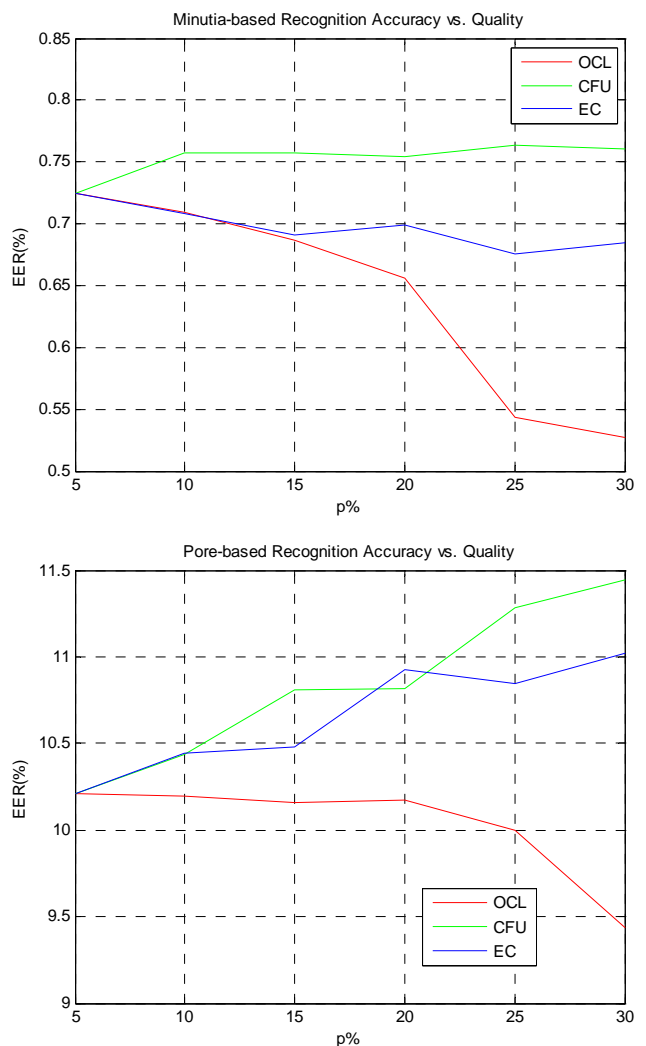

Fig. 3: The fingerprint recognition accuracy with respect to the quality of fingerprint images.

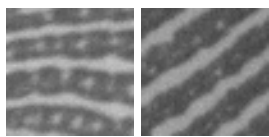

Fig. 4: Two example high resolution fingerprint fragments. The CFU index values of them are respectively 0.09 and 0.12 (from left to right). 
The EC quality index is almost effective when the minutia-based fingerprint matcher is used, but does not work for the pore-based fingerprint matcher. As discussed in Section 2, the EC quality index essentially evaluates the fingerprint ridge frequency field. Such frequency feature is important for the minutia extractor used here which is based on fingerprint images enhanced by Gabor filters, because the ridge frequency has to be estimated for the filters, and thus a good quality ridge frequency field is very important for the method. On the other hand, the pore extractor used in the experiments is less dependent on the ridge frequency. Moreover, the appearance of pores on ridges could bias the estimation of ridge frequency, and decrease the value of the EC quality index. Consequently, the EC quality index cannot well predict the recognition accuracy of pore-based fingerprint matcher as observed in Fig. 3.

Different from the other two quality indexes, the OCL quality index can well predict the recognition accuracy of both minutia-based and pore-based fingerprint matchers on high resolution fingerprint images. The ridge orientation field plays an important role in the extraction of both minutiae and pores. Fingerprint images with good quality ridge orientation field can thus benefit both minutia-based and pore-based fingerprint matchers. This is demonstrated by Fig. 3. Moreover, as small dots on ridges, the pores have little interference on the local dominant ridge orientation. Therefore, the OCL quality index still works well on high resolution fingerprint images and can well predict the recognition accuracy of fingerprint matchers when high resolution fingerprint images are used.

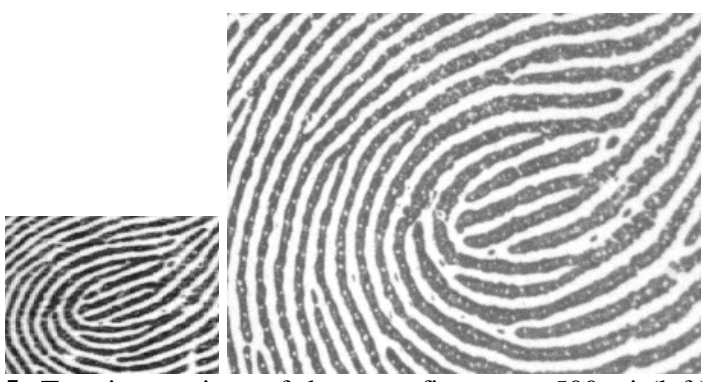

Fig. 5: Two impressions of the same finger at $\sim 500$ ppi (left) and $\sim 1200$ ppi (right).

\subsection{Discussion}

The above experiments clearly demonstrate that not all existing fingerprint image quality assessment methods are suitable for high resolution fingerprint images. This is due to the characteristics of high resolution fingerprint images compared with low resolution fingerprint images. As shown in Fig. 5, on high resolution fingerprint images, many fine features on ridges come into sight, e.g. pores (see the bright blobs on ridges). In conventional fingerprint matchers for low resolution fingerprint images, such fine ridge features are however taken as noise, rather than useful features, and hence will be suppressed by using some enhancement filters. As a result, many existing fingerprint image quality indexes cannot well handle high resolution fingerprint images as they are initially proposed for low resolution fingerprint images. One exception is the OCL quality index, which assesses the fingerprint image quality based on the ridge orientation field. This is because the orientation field composes the basis of fingerprint matchers for both low resolution and high resolution fingerprint images.

\section{CONSLUSION}

A comparative study has been presented in this paper for quality assessment of high resolution fingerprint images. The results show that not all existing image quality indexes are equally effective for high resolution fingerprint images when the fine ridge features (e.g. pores) on them are utilized in fingerprint recognition. Therefore, fingerprint image resolution and the specific features used for recognition have to be considered when designing fingerprint image quality indexes. In addition to the OCL quality index which is suitable for high resolution fingerprint images, we are going to develop new indexes specially designed for fine ridge features on high resolution fingerprint images.

\section{REFERENCES}

[1] D. Maltoni, D. Maio, A.K. Jain, and S. Prabhakar, Handbook of Fingerprint Recognition. $2^{\text {nd }}$ Edition. Springer, New York, 2009.

[2] A.K. Jain, Y. Chen, and M. Demirkus, "Pores and Ridges: High-Resolution Fingerprint Matching Using Level 3 Features," IEEE Trans. PAMI, vol. 29(1), pp. 15-27, 2007.

[3] Q. Zhao, D. Zhang, L. Zhang, and N. Luo, "High Resolution Partial Fingerprint Alignment Using Pore-Valley Descriptors," Pattern Recognition, vol. 43(3), pp. 1050-1061, 2010.

[4] M. Vatsa, R. Singh, A. Noore, and M.M. Houck, "Qualityaugmented Fusion of Level-2 and Level-3 Fingerprint Information using DSm Theory," International Journal of Approximate Reasoning, vol. 50(1), pp. 51-61, 2009.

[5] L. Shen, A.Kot, and W. Koo, "Quality Measures of Fingerprint Images,” AVBPA, pp. 266-271, 2001.

[6] E. Lim, X. Jiang, and W. Yau, "Fingerprint Quality and Validity Analysis,” ICIP, pp. 469-472, 2002.

[7] E. Lim, K. Toh, P. Suganthan, X. Jiang, and W. Yau, "Fingerprint Image Quality Analysis,” ICIP, pp. 1241-1244, 2004.

[8] Y. Chen, S. Dass, and A. Jain, "Fingerprint Quality Indices for Predicting Authentication Performance,” AVBPA, pp. 160-170, 2005.

[9] E. Tabassi and C. Wilson, "A Novel Approach to Fingerprint Image Quality,” ICIP, vol. 2, pp. 37-40, 2005.

[10] F. Alonso-Fernandez, J. Fiérrez-Aguilar, J. Ortega-Garcia, J. Gonzalez-Rodriguez, H. Fronthaler, K. Kollreider, and J. Bigün,

"A Comparative Study of Fingerprint Image-Quality Estimation Methods." IEEE Trans. IFS, vol. 2(4), pp. 734-743, 2007.

[11] J. Feng, "Combining Minutiae Descriptors for Fingerprint Matching," Pattern Recognition, vol. 41, pp. 342-352, 2008.

[12] Q. Zhao, L. Zhang, D. Zhang, N. Luo, and J. Bao, "Adaptive Pore Model for Fingerprint Pore Matching," ICPR, 2008.

[13] Q. Zhao, L. Zhang, D. Zhang, and N. Luo, "Direct Pore Matching for Fingerprint Recognition," ICB, pp. 597-606, 2009. 